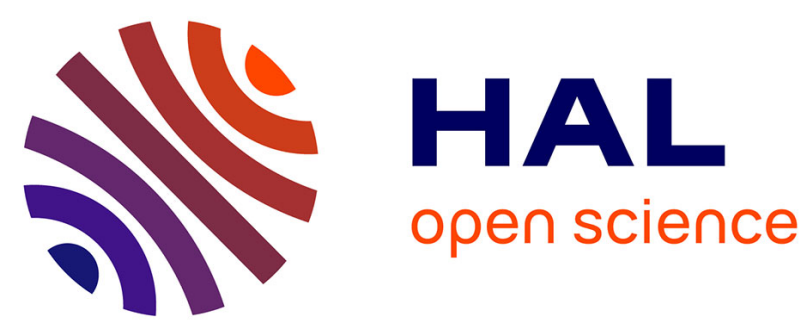

\title{
Cooperative Translation Control based on Consensus with Reference Velocity: a Source-seeking Application
}

\author{
Lara Briñon Arranz, Alexandre Seuret
}

\section{To cite this version:}

Lara Briñon Arranz, Alexandre Seuret. Cooperative Translation Control based on Consensus with Reference Velocity: a Source-seeking Application. ECC 2013 - 12th biannual European Control Conference, Jul 2013, Zurich, Switzerland. 6p. hal-00822715

\section{HAL Id: hal-00822715 https://hal.science/hal-00822715}

Submitted on 10 Jun 2013

HAL is a multi-disciplinary open access archive for the deposit and dissemination of scientific research documents, whether they are published or not. The documents may come from teaching and research institutions in France or abroad, or from public or private research centers.
L'archive ouverte pluridisciplinaire HAL, est destinée au dépôt et à la diffusion de documents scientifiques de niveau recherche, publiés ou non, émanant des établissements d'enseignement et de recherche français ou étrangers, des laboratoires publics ou privés. 


\title{
Cooperative Translation Control based on Consensus with Reference Velocity: a Source-seeking Application
}

\author{
Lara Briñón-Arranz ${ }^{1}$ and Alexandre Seuret ${ }^{2}$
}

\begin{abstract}
This paper deals with the design of cooperative control laws for nonlinear multi-agent systems. On the first hand, the control objectives are to ensure that a group of agents reaches a time-varying circular formation characterized by some external references representing the position of the center of the formation and its derivatives. In order to reduce the amount of information to be shared, the second part of the paper considers the situation where only the velocity of the center of the formation is available to each agent. Then a distributed consensus algorithm is provided in order to satisfy the same control objectives. Finally, an application to the source-seeking problem is proposed to emphasize previous contribution. These results are supported through computer simulations.
\end{abstract}

\section{INTRODUCTION}

Cooperative control problems and multi-agent systems have received a large attention in recent years. This field includes consensus algorithms [13], [19], flocking [12], formation control [5] and distributed estimation in sensor networks [8], [11], [22]. Engineering motivations for studying cooperative control approaches stem from increasing interest in groups of embedded systems, such as multi-vehicle and sensor networks.

A particularly relevant area deals with the formation control and the motion coordination, see [8], [9] and the references therein. For instance, cooperative control laws have been provided to make a fleet of agents obtain circular and parallel formations [8], [20]. Moving a formation of agents is pertinent to some applications where the agents should perform collaborative tasks requiring the formation to displace towards an a priori unknown direction. For instance, in source seeking applications, the formation is driven following the source gradient direction [11], [10]. The target tracking problem also requires consideration of timevarying formations. In these applications, the agents attempt encircling the target. Therefore, a circular formation whose center location is the target, seems very appropriate. Some cooperative approaches to carry out this challenge using a fleet of vehicles have been studied in the literature [6], [14]. As shown in [7], a circular formation can be useful to track the trajectory of a time-varying target.

The source localization of a signal distribution is a problem considered in recent literature [4], [11]. There are different approaches to deal with this topic, but the common

${ }^{1}$ L. Briñón-Arranz is with the Department of Automatic Control, GIPSALab, Grenoble, France. lara.brinon at gmail.com

${ }^{2}$ A. Seuret is with the CNRS, LAAS, 7 avenue du Colonel Roche and Universit de Toulouse, LAAS, F-31400 Toulouse, France. alexandre.seuret at laas.fr objective is to calculate the position of the source using measurements of the signal propagation. Several sourceseeking algorithms are based on gradient-descent methods. If it is available, the gradient of the signal strength can be used to produce a gradient-descent algorithm for a vehicle or group of vehicles [1]. A collaborative control law to steer the center of a circular formation of agents to the source of a signal distribution using only direct signal measurements is presented in [10]. An extension of this result is presented in [3] under a distributed approach. In this paper we apply a cooperative formation control law based on consensus algorithms to steer a group of vehicles to the source location assuming that the gradient corrupted by noise is available for all vehicles.

The paper is organized as follows. The following section presents the problem formulation introducing the model of the agents. In Section III a new translation control law is proposed. Section IV exposes a cooperative formation control design based on consensus with reference velocity algorithms. In Section V the source-seeking problem is tackled applying previous control laws to a group of agents. Finally, we will present our conclusions and future directions.

Notation. Let $\mathscr{G}=(V, E)$ be an undirected graph and $\mathbf{L}$ its Laplacian matrix. The set of agents is denoted by $V=\{1, \ldots, N\}$. Let $\mathscr{N}_{k}=\{j \in V:(k, j) \in E\}$ the set of neighbors of agent $k$. In the sequel, $\otimes$ denotes the Kronecker product and, for simplicity, we define $\overline{\mathbf{L}}=\mathbf{L} \otimes \mathbf{I}_{2}$ where $\mathbf{I}_{N} \in$ $\mathbb{R}^{N \times N}$ is the identity matrix. $\mathbf{R}_{\frac{\pi}{2}}=\left(\begin{array}{cc}0 & -1 \\ 1 & 0\end{array}\right)$ represents a rotation matrix through an angle $\frac{\pi}{2}$ counterclockwise around the origin.

\section{Problem formulation}

\section{A. Model of Agents}

Consider a group of $N$ identical vehicles modeled with unicycle kinematics subject to a simple non-holonomic constraint. The dynamics of agents is defined by:

$$
\begin{aligned}
\dot{x}_{k} & =v_{k} \cos \theta_{k} \\
\dot{y}_{k} & =v_{k} \sin \theta_{k} \\
\dot{\theta}_{k} & =u_{k}
\end{aligned}
$$

where $\mathbf{r}_{k}=\left(x_{k}, y_{k}\right)^{T} \in \mathbb{R}^{2}$ is the position vector of each agent $k=1, \ldots, N, \theta_{k}$ is its heading angle and $v_{k}, u_{k}$ are the control inputs. We consider here that each vehicle knows its absolute vector position $\mathbf{r}_{k}$ with respect to the inertial frame. 


\section{B. Control Objectives}

The main objective of this paper is to develop a cooperative control law to stabilize a group of vehicles to a circular formation which tracks a time-varying center.

In a first step, we consider that the center $\mathbf{c}(t)$ of the desired formation is a given external reference known for all the agents. In order to relax this assumption, the following step is to develop a distributed algorithm to reach an agreement on the center trajectory when the only information received by the agents is the velocity reference of the desired center of the formation. Based on a consensus algorithm with group reference velocity presented in [17], we design a cooperative translation control law that makes all agents converge to the same circular formation which tracks the given reference velocity.

Finally, we will present an application in the context of source localization. In this case, the group reference velocity is the gradient of the scalar field of interest. Applying the previous translation control law, a fleet of vehicles is able to reach an agreement on the trajectory of the formation center. Moreover, the formation tracks the given gradient direction of the scalar field. Thanks to this gradient information, the trajectory of the center steers the fleet to the source location.

\section{TRANSLATION OF A CIRCULAR MOTION}

The results presented in this paper establish a connection between our previous work dealing with translation control of a circular formation [2] and the source-seeking problem. First, we provide a new control law to make a group of agents converge to a circular motion which tracks the time-varying center $\mathbf{c}(t)$. The main difference with respect to [2] and thus a contribution of this paper is the new control strategy proposed. Using the convergence properties of an autonomous exosystem and a tracking control design, the new translation control law ensures robustness to uncertainties in the states' initial conditions $\mathbf{r}_{k}(0), \theta_{k}(0)$. The trajectory of the center $\mathbf{c}(t) \in \mathbb{R}^{2}$, is considered a given external reference. The radius of the circle $R$, and the rotation velocity $\omega_{0}$, are constant given parameters.

In order to exploit previous results dealing with circular motions from [15], [20], we introduce an exosystem represented by the following multi-agent dynamics:

$$
\begin{aligned}
\dot{\hat{x}}_{k} & =R\left|\omega_{0}\right| \cos \psi_{k} \\
\dot{\hat{y}}_{k} & =R\left|\omega_{0}\right| \sin \psi_{k} \\
\dot{\psi}_{k} & =\hat{u}_{k}
\end{aligned}
$$

where $\psi_{k}$ represents the angular orientation of the velocity vector $\dot{\hat{\mathbf{r}}}_{k}=\left(\dot{\hat{x}}_{k}, \dot{\hat{y}}_{k}\right)^{T}$ and $\hat{u}_{k}=f\left(\hat{\mathbf{r}}_{k}, \psi_{k}\right)$ is the control input of this autonomous exosystem. The following control law from [15] enforces the exosystem (2) to converge to a circular motion centered at the origin and with radius $R$ :

$$
\hat{u}_{k}=\omega_{0}\left(1+\kappa \dot{\hat{\mathbf{r}}}_{k}^{T} \hat{\mathbf{r}}_{k}\right)
$$

where $\kappa>0$ is a control parameter.

Our idea is to transform the exosystem circular trajectories via a translation by vector $\mathbf{c}(t)$. The resulting transformed trajectories will be considered as a reference to the multiagent states. The differences between the reference and the multi-agent state are defined by the following tracking error:

$$
\varepsilon_{k}=\mathbf{r}_{k}-\left(\hat{\mathbf{r}}_{k}+\mathbf{c}\right), \quad \forall k=1, \ldots, N
$$

In other words, our problem is to design control laws $v_{k}, u_{k}$ for the multi-agent system (1) such that the error $\varepsilon_{k}$ converges to zero.

In order to stabilize a fleet of agents to a time-varying circular motion, we propose a new control strategy. This new strategy follows three phases:

- Transformation: the desired formation is expressed as result of a translation applied to the unit circle and defined by a few number of parameters.

- Exosystem: we define a global stable exosystem (2) which converges, for instance, to a circular motion with fixed center, thanks to the beacon control law from [15].

- Tracking control design: we design the control inputs $\left(v_{k}, u_{k}\right)$ of the multi-agent system (1) in order to track the reference defined by the transformed circular trajectories of the exosystem, i.e., enforcing the error defined by (4) to converge to zero.

The following theorem presents the first contribution of this paper.

Theorem 1 Consider a twice differentiable function $c(t)$ : $\mathbb{R} \rightarrow \mathbb{R}^{2}$, with bounded first and second time-derivatives. Let $\omega_{0} \neq 0, \kappa>0, \alpha>0, \beta>0$ be four control parameters, $R>0$ be the radius of the desired circular motion and the following condition is satisfied:

$$
v_{k}>0 \quad \forall k=1, \ldots, N
$$

Then, for all initial conditions $\boldsymbol{r}(0), \theta(0)$, the control law

$$
\begin{aligned}
& \dot{v}_{k}=-\beta v_{k}+\frac{\dot{\boldsymbol{r}}_{k}^{T}}{v_{k}}\left[\hat{u}_{k} \boldsymbol{R} \frac{\pi}{2} \dot{\hat{\boldsymbol{r}}}_{k}+\ddot{\boldsymbol{c}}+\beta\left(\dot{\boldsymbol{r}}_{k}+\dot{\boldsymbol{c}}\right)+\alpha\left(\hat{\boldsymbol{r}}_{k}+\boldsymbol{c}-\boldsymbol{r}_{k}\right)\right] \\
& u_{k}=\frac{\hat{u}_{k} \dot{\boldsymbol{r}}_{k}^{T} \dot{\boldsymbol{r}}_{k}}{v_{k}^{2}}+\frac{\dot{\boldsymbol{r}}_{k}^{T} \boldsymbol{R}_{\frac{\pi}{2}}^{T}}{v_{k}^{2}}\left[\ddot{\boldsymbol{c}}+\beta\left(\dot{\hat{\boldsymbol{r}}}_{k}+\dot{\boldsymbol{c}}\right)+\alpha\left(\hat{\boldsymbol{r}}_{k}+\boldsymbol{c}-\boldsymbol{r}_{k}\right)\right]
\end{aligned}
$$

where $\dot{\hat{\boldsymbol{r}}}_{k}$ and $\hat{u}_{k}$ are respectively defined by (2) and (3), makes all the agents defined by (1) converge to a circular motion of radius $R$, and whose center tracks the time-varying reference $c(t)$. The direction of rotation is determined by the sign of $\omega_{0}$.

Proof: The first step is to ensure the convergence of the exosystem defined by (2) to a fixed circular motion. Consider following notation $\hat{\mathbf{r}}=\left(\hat{\mathbf{r}}_{1}^{T}, \ldots, \hat{\mathbf{r}}_{N}^{T}\right)^{T}$ and $\psi=\left(\psi_{1}, \ldots, \psi_{N}\right)^{T}$, and the following Lyapunov function:

$$
S(\hat{\mathbf{r}}, \psi)=\frac{1}{2} \sum_{k=1}^{N}\left\|\dot{\hat{\mathbf{r}}}_{k}-\omega_{0} \mathbf{R}_{\frac{\pi}{2}} \hat{\mathbf{r}}_{k}\right\|^{2} \geq 0
$$

At the equilibrium points of previous Lyapunov function, i.e., $S(\hat{\mathbf{r}}, \psi)=0$, the dynamics of the exosystem (2) satisfies $\dot{\hat{\mathbf{r}}}_{k}-\omega_{0} \mathbf{R}_{\frac{\pi}{2}} \hat{\mathbf{r}}_{k}=0$. Thus, the position vector and its velocity vector are perpendicular, i.e., $\dot{\mathbf{r}}_{k}^{T} \hat{\mathbf{r}}_{k}=0$. This condition leads to the kinematic relation for the rotation of the rigid body, 
i.e., the vectors $\hat{\mathbf{r}}_{k}$ are turning around the frame origin at the equilibrium. Evaluating the derivative of $S(\hat{\mathbf{r}}, \psi)$ along the solutions of the resulting closed-loop system (2) and considering the proposed control law (3) leads to:

$$
\begin{aligned}
\dot{S}(\hat{\mathbf{r}}, \psi) & =\sum_{k=1}^{N}\left(\hat{u}_{k} \mathbf{R}_{\frac{\pi}{2}} \dot{\mathbf{r}}_{k}-\omega_{0} \mathbf{R}_{\frac{\pi}{2}} \dot{\mathbf{r}}_{k}\right)^{T}\left(\dot{\hat{\mathbf{r}}}_{k}-\omega_{0} \mathbf{R}_{\frac{\pi}{2}} \hat{\mathbf{r}}_{k}\right) \\
& =\sum_{k=1}^{N} \omega_{0} \hat{\mathbf{r}}_{k}^{T} \dot{\mathbf{r}}_{k}\left(\omega_{0}-\hat{u}_{k}\right)=-\kappa \sum_{k=1}^{N}\left(\omega_{0} \hat{\mathbf{r}}_{k}^{T} \dot{\mathbf{r}}_{k}\right)^{2} \leq 0
\end{aligned}
$$

In conclusion, $S(\hat{\mathbf{r}}, \psi)$ is a suitable Lyapunov function for exosystem (2), and by the LaSalle Invariance Principle, the solutions converge to the largest invariant set $\Lambda$, for which $\dot{S}=0$ and consequently, the dynamics of the exosystem satisfy $\dot{\mathbf{r}}_{k}=\omega_{0} \mathbf{R} \frac{\pi}{2} \hat{\mathbf{r}}_{k}$ which corresponds to a circular motion.

The second step corresponds to the tracking control design. In order to achieve the objective $\mathbf{r}_{k} \rightarrow \hat{\mathbf{r}}_{k}+\mathbf{c}$, the tracking error is defined by (4). With a view to make the error converge to zero, we wish to impose the error dynamics

$$
\ddot{\varepsilon}_{k}=-\beta \dot{\varepsilon}_{k}-\alpha \varepsilon_{k}
$$

where $\alpha>0, \beta>0$. Thus, the error converges exponentially to zero. The dynamics of the error equation determines the control law for the original system (1) since:

$$
\ddot{\varepsilon}_{k}=\ddot{\mathbf{r}}_{k}-\ddot{\hat{\mathbf{r}}_{k}}-\ddot{\mathbf{c}}=\frac{\dot{v}_{k}}{v_{k}} \dot{\mathbf{r}}_{k}+u_{k} \mathbf{R}_{\frac{\pi}{2}} \dot{\mathbf{r}}_{k}-\hat{u}_{k} \mathbf{R}_{\frac{\pi}{2}} \dot{\hat{\mathbf{r}}}_{k}-\ddot{\mathbf{c}}
$$

According to the error dynamics and multiplying this last equation by $\dot{\mathbf{r}}_{k}^{T}$ and by $\dot{\mathbf{r}}_{k}^{T} \mathbf{R}_{\frac{\pi}{2}}^{T}$ both following expressions hold:

$$
\begin{aligned}
& \dot{v}_{k} v_{k}=-\beta v_{k}^{2}+\dot{\mathbf{r}}_{k}^{T}\left[\hat{u}_{k} \mathbf{R}_{\frac{\pi}{2}} \dot{\hat{\mathbf{r}}}_{k}+\ddot{\mathbf{c}}+\beta\left(\dot{\hat{\mathbf{r}}}_{k}+\dot{\mathbf{c}}\right)-\alpha\left(\mathbf{r}_{k}-\hat{\mathbf{r}}_{k}-\mathbf{c}\right)\right] \\
& u_{k} v_{k}^{2}=\hat{u}_{k} \dot{\mathbf{r}}_{k}^{T} \dot{\hat{\mathbf{r}}}_{k}+\dot{\mathbf{r}}_{k}^{T} \mathbf{R}_{\frac{\pi}{2}}^{T}\left[\ddot{\mathbf{c}}+\beta\left(\dot{\mathbf{r}}_{k}+\dot{\mathbf{c}}\right)-\alpha\left(\mathbf{r}_{k}-\hat{\mathbf{r}}_{k}-\mathbf{c}\right)\right]
\end{aligned}
$$

By definition, this control law enforces exponential convergence of the tracking error dynamics away from the singularity $v_{k}=0$. If condition (5) is satisfied then, the control inputs of (6) are respectively obtained.

Thanks to previous definition of the error both following equations hold when $t \rightarrow \infty, \mathbf{r}_{k}=\hat{\mathbf{r}}_{k}+\mathbf{c}$ and $\dot{\mathbf{r}}_{k}=\dot{\mathbf{r}}_{k}+\dot{\mathbf{c}}$. Taking into account the circular control law (3), the exosystem converges to $\dot{\mathbf{r}}_{k}=\omega_{0} \mathbf{R} \frac{\pi}{2} \hat{\mathbf{r}}_{k}$, hence, the agents converge to a time-varying circular motion since, for all $k=1, \ldots, N$ :

$$
\dot{\mathbf{r}}_{k}=\underbrace{\omega_{0} \mathbf{R} \frac{\pi}{2}\left(\mathbf{r}_{k}-\mathbf{c}\right)}_{\text {circular motion }}+\underbrace{\dot{\mathbf{c}}}_{\text {translation }}
$$

Note that Theorem 1 presents a dynamic control law in which the control inputs are $\left(\dot{v}_{k}, u_{k}\right)$. As mentioned above, this control law is robust to uncertainties in the initial conditions of the agents' states which was not the case in [2].

Remark 1 Note that we can also consider a cooperative control law for the exosystem in order to distribute uniformly the agents along the formation. Therefore, previous control law (6) where $\dot{\hat{\boldsymbol{r}}}_{k}$ is defined by (2) with

$$
\left\{\begin{array}{l}
\hat{u}_{k}=\omega_{0}\left(1+\kappa \dot{\hat{\boldsymbol{r}}}_{k}^{T} \hat{\boldsymbol{r}}_{k}\right)-\frac{\partial U}{\partial \psi_{k}} \\
U(\psi)=\frac{K}{N} \sum_{m=1}^{\lfloor N / 2\rfloor} \frac{1}{2 m^{2}} \boldsymbol{B}_{m} \overline{\boldsymbol{L}} \boldsymbol{B}_{m}
\end{array}\right.
$$

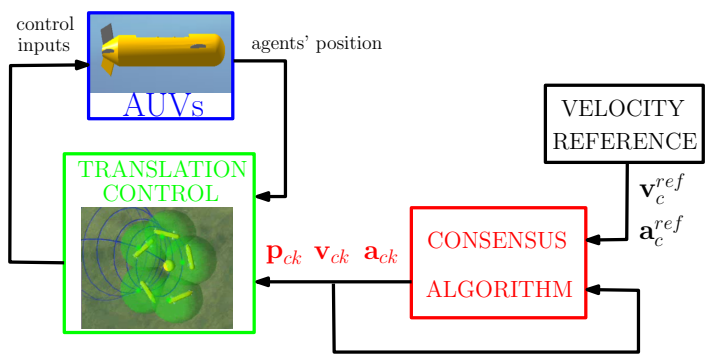

Fig. 1. Control strategy: consensus with a reference velocity

where $\boldsymbol{B}_{m}=\left(\cos m \psi_{k}, \sin m \psi_{k}, \ldots, \cos m \psi_{N}, \sin m \psi_{N}\right)^{T}$, makes all the agents defined by (1) converge to a timevarying circular formation of radius $R$ and center $\boldsymbol{c}(t)$. Moreover, for $K>0$, the agents are distributed uniformly along the circular formation, see [20].

\section{COOPERATIVE TRANSLATION CONTROL DESIGN}

The final application aims to solve the source-seeking problem taking into account communication constraints. In this context, the agents must be able to collaborate in order to decide the trajectory of the formation center. Therefore, the idea now is to implement a cooperative algorithm to make the fleet of vehicles converge to the same circular formation considering that the reference of the center $\mathbf{c}(t)$ is unknown. The approach presented in this section considers that each agent only knows the first and second derivatives of the center which defines the time-varying circular formation. In this situation, a consensus protocol is designed to reach the same center trajectory $\mathbf{c}(t)$ for all the agents. The given reference is thus, the desired velocity of the center formation. The objective is for the agents to reach the same circular formation, i.e., to reach a consensus on the center of the circle.

Consensus problems with a reference velocity are already studied in [16], [17], [21] for double-integrator dynamics. In the context of a moving circle, the velocity of the center is a given reference denoted by $\mathbf{v}_{c}^{r e f} \in \mathbb{R}^{2}$ known to all the vehicles. Besides, the acceleration represented by $\mathbf{a}_{c}^{\text {ref }} \in \mathbb{R}^{2}$ is also known to all the vehicles. Nevertheless, the trajectory of the center is not defined. This is coherent with a sourceseeking situation in which the gradient of the scalar field of interest is the desired velocity of the formation center. This information could be a given reference for the agents, but the center of the circular formation is not a known parameter. In this situation, each agent computes its own estimated position of the center of the circular formation represented by $\mathbf{p}_{c k} \in \mathbb{R}^{2}$, its own estimated velocity of the center denoted by $\mathbf{v}_{c k} \in \mathbb{R}^{2}$ and its own estimated acceleration of the center represented by $\mathbf{a}_{c k} \in \mathbb{R}^{2}$.

In order to keep the formation, the position of the center calculated for all the vehicles must be the same. Summarizing, the proposed control strategy, which is explained schematically in Fig. 1, is composed by the following steps:

1) Each agent computes its own estimated position of the center and its derivatives. 
2) A distributed algorithm is implemented to reach consensus on the position of the formation center using the external references $\mathbf{v}_{c}^{r e f}, \mathbf{a}_{c}^{r e f}$.

3) The inputs of the translation control law for each agent are obtained from the consensus algorithm.

\section{A. Consensus with reference velocity}

In order to fulfill the previous items, a new dynamic variable representing the center of the circle computed by each agent is introduced and satisfies the following dynamics:

$$
\begin{aligned}
\dot{\mathbf{p}}_{c k} & =\mathbf{v}_{c k} \\
\dot{\mathbf{v}}_{c k} & =\mathbf{a}_{c k}
\end{aligned}
$$

Consensus is reached for (10) if for all $\mathbf{p}_{c k}(0)$ and $\mathbf{v}_{c k}(0)$, then $\mathbf{p}_{c k}(t) \rightarrow \mathbf{p}_{c j}(t)$ and $\mathbf{v}_{c k}(t) \rightarrow \mathbf{v}_{c}^{r e f}(t)$ asymptotically as $t \rightarrow \infty$. Based on [17], [18], we propose the following consensus algorithm with a group reference velocity:

$$
\mathbf{a}_{c k}=\mathbf{a}_{c}^{r e f}-\gamma\left(\mathbf{v}_{c k}-\mathbf{v}_{c}^{r e f}\right)-\sum_{j \in \mathscr{N}_{k}}\left(\mathbf{p}_{c k}-\mathbf{p}_{c j}\right)
$$

where $\gamma$ is a positive gain. In [17] it is shown that this consensus algorithm converges when the directed communication graph has a spanning tree. This is a generalization of the results presented in [16] for directed graphs. In this paper, the communication between the vehicles is considered undirected, such that the Laplacian matrix of the communication graph is always symmetric and all its eigenvalues are real and nonnegative. In consequence, the result from [17] can be rewritten for undirected graphs as follows:

Theorem 2 (Ren 2008 [17]) Consider the consensus algorithm (11), if the undirected communication graph between the agents is connected and $\boldsymbol{\gamma}>0$ then $\boldsymbol{p}_{c k}(t) \rightarrow \boldsymbol{p}_{c j}(t)$ and $\boldsymbol{v}_{c k}(t) \rightarrow \boldsymbol{v}_{c}^{\text {ref }}(t)$ asymptotically as $t \rightarrow \infty$ for all $k, j$.

Proof: The details of the proof can be found in [17] for the general case of a directed graph. Rewriting the consensus algorithm with a group reference velocity in a vector form, previous equation (11) becomes:

$$
\mathbf{a}_{c}=\mathbf{1} \otimes \mathbf{a}_{c}^{r e f}-\gamma\left(\mathbf{v}_{c}-\mathbf{1} \otimes \mathbf{v}_{c}^{r e f}\right)-\mathbf{L} \mathbf{p}_{c}
$$

where $\mathbf{p}_{c}=\left(\mathbf{p}_{c 1}^{T}, \ldots, \mathbf{p}_{c N}^{T}\right)^{T}$ represents the vector of all the local positions of the center computed by each agent (analogically the vectors $\mathbf{v}_{c}$ and $\left.\mathbf{a}_{c}\right)$ and $\mathbf{1}=(1, \ldots, 1)^{T} \in \mathbb{R}^{N}$ is the vector of ones. Considering that $\mathbf{p}_{c}^{\text {ref }}$ represents the reference trajectory associated to the given $\mathbf{v}_{c}^{r e f}$, a new variable $\chi=$ $\left(\chi_{1}, \ldots, \chi_{N}\right)^{T}$ is introduced to express the following error equation $\chi=\mathbf{p}_{c}-\mathbf{1} \otimes \mathbf{p}_{c}^{\text {ref }}$. By definition, the vector of ones $\mathbf{1}$ is always a right eigenvector of the Laplacian matrix $\mathbf{L}$ corresponding to the eigenvalue 0 . Using this property, the previous compact form of the consensus algorithm (12) can be rewritten as $\ddot{\chi}=-\gamma \dot{\chi}-\mathbf{L} \chi$ which refers to a doubleintegrator consensus algorithm. In conclusion, if the communication graph $\mathscr{G}$ is connected, for all $\chi_{k}(0)$ and $\dot{\chi}_{k}(0)$, then $\chi_{k}(t) \rightarrow \chi_{j}(t)$ and $\dot{\chi}_{k}(t) \rightarrow 0$ asymptotically as $t \rightarrow \infty$. Therefore, for all $\mathbf{p}_{c k}(0)$ and $\mathbf{v}_{c k}(0), \mathbf{p}_{c k}(t) \rightarrow \mathbf{p}_{c j}(t)$ and $\mathbf{v}_{c k}(t) \rightarrow \mathbf{v}_{c}^{r e f}(t)$ asymptotically as $t \rightarrow \infty$.

\section{B. Cooperative Translation Control Design}

This previous consensus algorithm allows the agents to reach an agreement on the trajectory of the center of the circular formation from a given reference velocity known to all the agents. The center trajectory and its first and second derivatives computed for each agent will be the inputs of the translation control law presented in previous section. In this situation, the error is now defined by

$$
\varepsilon_{k}=\mathbf{r}_{k}-\left(\hat{\mathbf{r}}_{k}+\mathbf{p}_{c k}\right) \quad \forall k=1, \ldots, N
$$

where the dynamics of the exosystem $\dot{\hat{\mathbf{r}}}_{k}$ are defined by (2) and the closed-loop dynamics are imposed by the control law (3). Therefore, the previous control law (6) developed to move the center of a circular formation following a given reference becomes:

$$
\dot{v}_{k}=-\beta v_{k}+\frac{\dot{\mathbf{r}}_{k}^{T}}{v_{k}}\left[\hat{u}_{k} \mathbf{R}_{\frac{\pi}{2}} \dot{\hat{\mathbf{r}}}_{k}+\mathbf{a}_{c k}+\beta\left(\dot{\hat{\mathbf{r}}}_{k}+\mathbf{v}_{c k}\right)+\alpha\left(\hat{\mathbf{r}}_{k}+\mathbf{p}_{c k}-\mathbf{r}_{k}\right)\right]
$$

$$
u_{k}=\frac{\hat{u}_{k} \dot{\mathbf{r}}_{k}^{T} \dot{\mathbf{r}}_{k}}{v_{k}^{2}}+\frac{\dot{\mathbf{r}}_{k}^{T} \mathbf{R}_{\frac{\pi}{2}}^{T}}{v_{k}^{2}}\left[\mathbf{a}_{c k}+\beta\left(\dot{\mathbf{r}}_{k}+\mathbf{v}_{c k}\right)+\alpha\left(\hat{\mathbf{r}}_{k}+\mathbf{p}_{c k}-\mathbf{r}_{k}\right)\right]
$$

where the position of the center computed for agent $k$ and its velocity and acceleration are $\mathbf{p}_{c k}, \mathbf{v}_{c k}$ and $\mathbf{a}_{c k}$ respectively, obtained from the consensus algorithm (11). To formalize this new collaborative approach the following corollary holds.

Corollary 1 Let $\boldsymbol{v}^{\text {ref }}$ and $\boldsymbol{a}^{\text {ref }}$ be the velocity and acceleration references of the desired center formation. Let $R$ be the desired radius of the circular formation, $\omega_{0} \neq 0, \kappa>0, \beta>$ $0, \alpha>0$ and $\gamma>0$ be five control parameters and condition (5) is satisfied. Then the control law (13) makes each agent defined by (1) converge to a circular motion with radius $R$ and time-varying center $\boldsymbol{p}_{c k}$. Thanks to the consensus algorithm (11) applied to the center dynamics (10), if the undirected communication graph is connected then all the centers reach a consensus asymptotically and their velocities follow the reference velocity $\boldsymbol{v}^{\text {ref }}$. The direction of rotation is determined by the sign of $\omega_{0}$.

Proof: As it is shown in Fig. 1, the whole system consists of two uncoupled systems. The first one is composed of the dynamics of the multi-agent system (1) and the translation control law (13). Thanks to Theorem 1, this control law (13) makes each agent converge to a circular motion whose center is the time-varying reference $\mathbf{p}_{c k}$.

The second system represents a consensus algorithm which is implemented to reach an agreement on the center of the formation for all the agents. Thanks to Theorem 2, the collaborative algorithm (11) makes system (10) reach consensus and therefore, asymptotically, all the computed centers satisfy $\mathbf{p}_{c k}=\mathbf{p}_{c j}=\mathbf{c}_{0} \quad \forall k, j$. Consequently, all the vehicles describe a circular motion following the timevarying center $\mathbf{c}_{0}$.

\section{Consensus with reference velocity corrupted by noise}

Previous collaborative algorithm can be improved by considering that each agent receives the external reference 


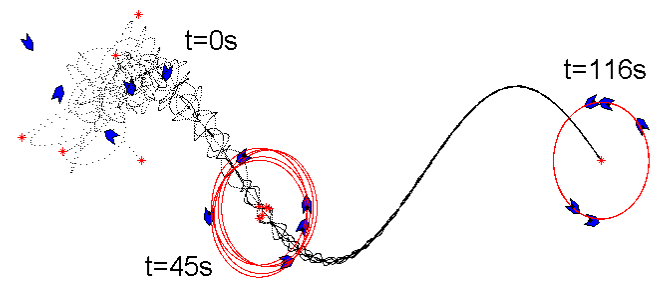

Fig. 2. Simulation of five agents governed by the control law (13) where the center computed by each agent results from the consensus algorithm (11). The black lines represent the centers' trajectories.

velocity corrupted by noise. It is assumed that the external reference received for each agent $k$ is then $\mathbf{s}_{k}(t)=\mathbf{v}_{c}^{r e f}+\mathbf{w}_{v k}$, where $\mathbf{w}_{v k} \in \mathbb{R}^{2}$ is a vector whose components are Gaussian zero-mean noise.

Intuitively, if the noise is bounded then, the previous algorithm reaches consensus in a closed ball centered at the consensus final value, as will be shown in simulation. However, the mathematical details of the proof of this proposition will be analyzed formally in future works.

\section{Simulation results}

The consensus algorithm (11) is implemented to generate the reference of the center circular formation in order to apply the translation control law (13). The group reference velocity is given by $\mathbf{v}_{c}^{r e f}=(0.2,0.24 \cos 0.08 t)^{T}$ and the initial conditions of the position of the center are different for each agent.

Fig. 2 shows a simulation of five agents governed by the translation control law (13) with the consensus algorithm (11) to provide the reference of the center of the circular formation. The control parameters are $R=2, \omega_{0}=\kappa=\alpha=$ $\beta=1$ and $\gamma=0.1$. The communication graph is a ring (circulant graph), therefore is connected. The figure shows three snapshots, the initial conditions, and two states for $t=45 s$ and $t=116 s$. The red circles represent the circular motion corresponding to each agent at each instant. The black lines represent the trajectories of each estimated center. This simulation shows that the center of each agent achieve consensus, then the common center tracks the given reference velocity and the circular formation is maintained.

Fig. 3 displays the evolution of the centers' trajectories $\mathbf{p}_{c k}$ and the centers' velocities $\mathbf{v}_{c k}$ computed by each agent $k$ for the same simulation of five agents. Starting from any initial condition, the collaborative algorithm (11) makes the agents reach consensus on the center position and all the centers' velocities converge asymptotically to the external reference.

\section{SOURCE-SEEKING VIA A CIRCULAR FORMATION}

This section presents the application of previous formation control law based on consensus with reference velocity to the source-seeking problem. The objective now is to steer a circular formation of unicycle-like modeled vehicles to the source location of a signal. In mathematical terms, the signal distribution emitted by the source is a spacial function
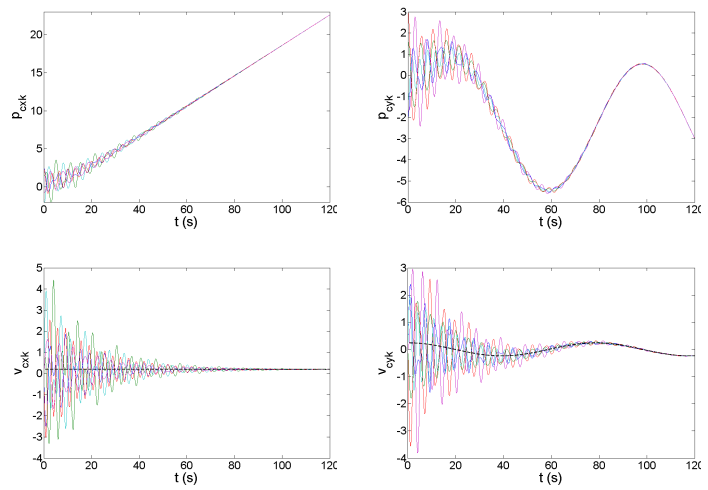

Fig. 3. Evolution of the centers' positions and velocities $\boldsymbol{p}_{c k}=\left(p_{c x k}, p_{c y k}\right)^{T}$, $\boldsymbol{v}_{c k}=\left(v_{c x k}, v_{c y k}\right)^{T}$ corresponding to the previous simulation of five agents shown in Fig. 2.

representing the scalar field with a maximum in the position where the source is located. For instance, the source could be a point of chemical contamination and the signal would be that chemical's concentration in the environment. We consider here that the signal is emitted by a single source such that the source is the only maximum of the scalar field. The signal distribution is assumed to decay away from the position of the source. The scalar field with elliptical level curves is given by

$$
\sigma(x, y)=10 e^{-\left(x^{2} / 10^{3}+y^{2} / 200\right)}
$$

whose maximum is located at $(0,0)$.

Using a gradient-descent algorithm the group of agents can be driven to the source of the the signal distribution, see [1], [10]. In this paper we consider that the gradient of the signal distribution is kwnon for all the agents. Our approach considers this gradient information as a reference velocity for the group of vehicles. Let $\nabla \sigma(\mathbf{p})$ denote the gradient of function $\sigma$ at position $\mathbf{p} \in \mathbb{R}^{2}$. We assume that the ASV estimates the gradient at the center of mass of the vehicles, such that $\nabla \sigma\left(\mathbf{p}_{c m}\right)$. Therefore, the reference velocity for the circular formation becomes $\mathbf{v}_{c}^{\text {ref }}=\nabla \sigma\left(\mathbf{p}_{c m}\right)^{T}$. Each agent $k$ receives this reference corrupted by a Gaussian white noise $\mathbf{w}_{v k}$. The aim to the formation is to track this reference. Applying the consensus algorithm (11), the agents reach an agreement on the center trajectory and thanks to control law (13) the circular formation is driven to the source location.

Fig. 4 shows a simulation of six agents governed by the control law (13) where the center of the formation computed by each agent results from the consensus algorithm (11) and the velocity reference is the gradient of the scalar field corrupted by noise, as explained before. The control parameters are $R=2, \omega_{0}=\kappa=\beta=\alpha=1$ and $\gamma=0.1$. The communication graph is a ring, therefore is connected. The figure shows three snapshots: the void agents corresponds to the initial conditions and the red ones to two different states at $t=25 \mathrm{~s}$ and at $t=250 \mathrm{~s}$. The blue lines represent the trajectories of each computed center $\mathbf{p}_{c k}$ which are described by the red stars. The fleet of agents reaches a consensus on 


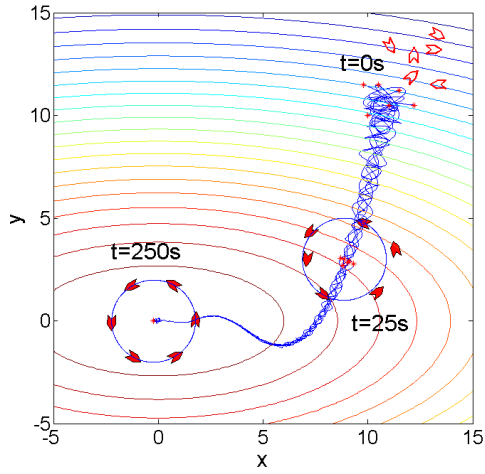

Fig. 4. Cooperative gradient-descent with a fleet of six AUVs
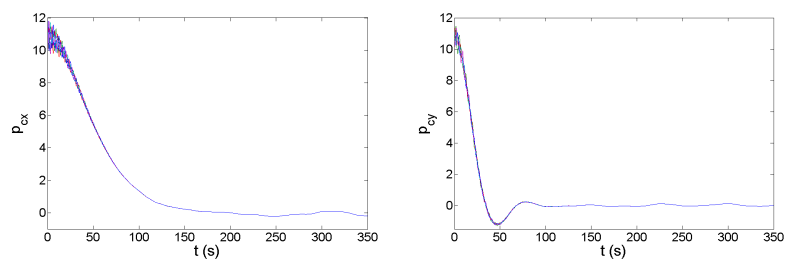

Fig. 5. Evolution of the centers' positions $\boldsymbol{p}_{c k}$ components corresponding to the previous simulation of six agents shown in Fig. 4.

the center of the formation and thanks to the gradient-descent strategy the formation is steered to the source location.

Fig. 5 displays the evolution of the trajectories of centers $\mathbf{p}_{c k}$ computed by each agent $k$ for the same simulation of six agents. As expected, the agents reach a consensus on the centers and the common center reaches exactly the source location. Note that if the gradient information is corrupted by noise, the formation is driven to neighborhood of the source position.

\section{CONCLUSION AND FUTURE WORKS}

This paper provides a new cooperative control design to stabilize a fleet of agents to a time-varying circular formation. First, the translation control law presented here makes the agents converge to a circular motion whose center tracks a given time-varying reference. In order to relax this assumption, a cooperative control law based on consensus algorithms with a group reference velocity is proposed to reach an agreement on the center formation while tracking a reference velocity. This approach is applied in the context of source localization. In this case, we consider that the gradient of the scalar field is known for all the agents. The communication constraints between the vehicles and the problem of noise are taken into account.

Future works will be focused on improving previous results dealing with a reference velocity corrupted by noise. A future direction is the extension of this work to decentralized algorithms using the signal measurements of the agents to estimate the gradient direction of the scalar field following our previous result from [3].

\section{ACKNOWLEDGMENTS}

We would like to thank Rodolphe Sepulcre and Carlos Canudas de Wit for their useful comments and interesting remarks.

\section{REFERENCES}

[1] R. Bachmayer and N. E. Leonard. Vehicle networks for gradient descent in a sampled environment. In Proc. of the 41st IEEE Conference on Decision and Control, 2002.

[2] L. Briñón-Arranz, A. Seuret, and C. Canudas-de-Wit. Translation control of a fleet circular formation of AUVs under finite communication range. In Proc. of the 48th IEEE Conference on Decision and Control, 2009.

[3] L. Briñón-Arranz, A. Seuret, and C. Canudas-de-Wit. Collaborative estimation of gradient direction by a formation of AUVs under communication constraints. In Proc. of the 50th IEEE Conference on Decision and Control, 2011.

[4] J. Cochran and M. Krstić. Nonholonomic source seeking with tuning of angular velocity. IEEE Trans. on Automatic Control, 54(4):717731, 2009.

[5] M. Egerstedt and X. Hu. Formation constrained multi-agent control. IEEE Trans. on Robotics and Automation, 17:947-951, 2001.

[6] D. J. Klein and K. A. Morgansen. Controlled collective motion for trajectory tracking. In Proc. of the 2006 American Control Conference, 2006.

[7] Y. Lan, Z. Lin, M. Cao, and G. Yan. A distributed reconfigurable control law for escorting and patrolling missions using teams of unicycles. In Proc. of the 49th IEEE Conference on Decision and Control, 2010.

[8] N. E. Leonard, D. A. Paley, F. Lekien, R. Sepulchre, D. M. Frantatoni, and R. E. Davis. Collective motion, sensor networks and ocean sampling. Proc. of the IEEE, 95:48-74, 2007.

[9] S. Martínez, J. Cortés, and F. Bullo. Motion coordination with distributed information. IEEE Control Systems Magazine, 27:75-88, 2007.

[10] B. J. Moore and C. Canudas-de-Wit. Source seeking via collaborative measurements by a circular formation of agents. In Proc. of the 2010 IEEE American Control Conference, 2010.

[11] P. Ogren, E. Fiorelli, and N. E. Leonard. Cooperative control of mobile sensor networks: Adaptive gradient climbing in a distributed environment. IEEE Trans. on Automatic Control, 49:1292-1302, 2004.

[12] R. Olfati-Saber. Flocking for multi-agent dynamic systems: Algorithms and theory. IEEE Trans. on Automatic Control, 51:401-420, 2006.

[13] R. Olfati-Saber and R. M. Murray. Consensus problems in networks of agents whit switching topology and time-delays. IEEE Trans. on Automatic Control, 2004.

[14] D. A. Paley, N. E. Leonard, and R. Sepulchre. Collective motion: Bistability and trajectory tracking. In Proc. of the 43th IEEE Conference Decision and Control, 2004.

[15] D. A. Paley, N. E. Leonard, and R. Sepulchre. Oscillator models and collective motion: Splay state stabilization of self-propelled particles. In Proc. of the 44th IEEE Conference on Decision and Control, 2005.

[16] W. Ren. Consensus strategies for cooperative control of vehicle formations. IET Control Theory \& Applications, 1(2):505-512, 2007.

[17] W. Ren. On consensus algorithms for double-integrator dynamics. IEEE Trans. on Automatic Control, 2008.

[18] W. Ren and R. W. Beard. Distributed Consensus in Multi-vehicle Cooperative Control. Communication and Control Engineering. Springer, 2007.

[19] W. Ren, R. W. Beard, and E. M. Atkins. A survey of consensus problems in multi-agent coordination. In Proc. of the 2005 American Control Conference, 2005.

[20] R. Sepulchre, D. A. Paley, and N. E. Leonard. Stabilization of planar collective motion with limited communication. IEEE Trans. on Automatic Control, 53:706-719, 2008.

[21] G. Xie and L. Wang. Consensus control for a class of networks of dynamic agents. International Journal of Robust and Nonlinear Control, 17(10-11):941-959, 2007.

[22] F. Zhang and N. E. Leonard. Cooperative filters and control for cooperative exploration. IEEE Trans. on Automatic Control, 55:650$663,2010$. 